\title{
Brain Circuits Mediating Opposing Effects on Emotion and Pain
}

\author{
You-Qing Cai, ${ }^{1}$ Wei Wang, ${ }^{1}$ Adriana Paulucci-Holthauzen, ${ }^{2}$ and $®$ Zhizhong Z. Pan ${ }^{1}$ \\ Departments of ${ }^{1}$ Anesthesiology and Pain Medicine and ${ }^{2}$ Genetics, The University of Texas M. D. Anderson Cancer Center, Houston, Texas 77030
}

The amygdala is important for processing emotion, including negative emotion such as anxiety and depression induced by chronic pain. Although remarkable progress has been achieved in recent years on amygdala regulation of both negative (fear) and positive (reward) behavioral responses, our current understanding is still limited regarding how the amygdala processes and integrates these negative and positive emotion responses within the amygdala circuits. In this study with optogenetic stimulation of specific brain circuits, we investigated how amygdala circuits regulate negative and positive emotion behaviors, using pain as an emotional assay in male rats. We report here that activation of the excitatory pathway from the parabrachial nucleus (PBN) that relays peripheral pain signals to the central nucleus of amygdala $(\mathrm{CeA})$ is sufficient to cause behaviors of negative emotion including anxiety, depression, and aversion in normal rats. In strong contrast, activation of the excitatory pathway from basolateral amygdala (BLA) that conveys processed corticolimbic signals to CeA dramatically opposes these behaviors of negative emotion, reducing anxiety and depression, and induces behavior of reward. Surprisingly, activating the PBN-CeA pathway to simulate pain signals does not change pain sensitivity itself, but activating the BLA-CeA pathway inhibits basal and sensitized pain. These findings demonstrate that the pain signal conveyed through the PBN-CeA pathway is sufficient to drive negative emotion and that the corticolimbic signal via the BLA-CeA pathway counteracts the negative emotion, suggesting a top-down brain mechanism for cognitive control of negative emotion under stressful environmental conditions such as pain.

Key words: amygdala; brain circuits; emotion; optogenetics; pain

\section{Significance Statement}

It remains unclear how the amygdala circuits integrate both negative and positive emotional responses and the brain circuits that link peripheral pain to negative emotion are largely unknown. Using optogenetic stimulation, this study shows that the excitatory projection from the parabrachial nucleus to the central nucleus of amygdala (CeA) is sufficient to drive behaviors of negative emotion including anxiety, depression, and aversion in rats. Conversely, activation of the excitatory projection from basolateral amygdala to CeA counteracts each of these behaviors of negative emotion. Thus, this study identifies a brain pathway that mediates pain-driven negative emotion and a brain pathway that counteracts these emotion behaviors in a top-down mechanism for brain control of negative emotion.

\section{Introduction}

Amygdala is an important part of the brain's corticolimbic network that performs evaluation, cognition, and memory of emotion-

Received Sept. 26, 2017; revised May 23, 2018; accepted May 29, 2018.

Author contributions: Y.-Q.C. wrote the first draft of the paper; Z.Z.P. edited the paper. Y.-Q.C., W.W., and Z.Z.P. designed research; Y.-Q.C., W.W., and A.P.-H. performed research; Y.-Q.C., W.W., A.P.-H., and Z.Z.P. analyzed data; Y.-Q.C. and Z.Z.P. wrote the paper.

This work was supported by the NIH National Institute of Dental and Craniofacial Research Grant DE025943. We thank Dr. Karl Deisseroth for providing and permitting the use of various viral vectors and the Vector Core Facility at The University of North Carolina for help in preparing the vectors.

The authors declare no competing financial interests.

Correspondence should be addressed to Dr. Zhizhong Z. Pan, Department of Anesthesiology and Pain Medicine,

The University of Texas M. D. Anderson Cancer Center, 1515 Holcombe Boulevard, Unit 110, Houston, TX 77030. E-mail:zzpan@mdanderson.org.

DOI:10.1523/JNEUROSCI.2780-17.2018

Copyright $\odot 2018$ the authors $\quad 0270-6474 / 18 / 386340-10 \$ 15.00 / 0$ related environmental inputs, and regulates decision-making and behavioral response (Vogt, 2005; Shackman et al., 2011; Baliki and Apkarian, 2015). The amygdala primarily regulates and mediates emotion-related learning behaviors including both negative emotional responses, such as fear and pain, and positive emotional responses, such as drug reward (Baxter and Murray, 2002; Gottfried et al., 2003; Zald, 2003; Murray, 2007; Neugebauer, 2015). This process is regulated by multiple amygdala nuclei and is well represented by the signaling pathway from the basolateral amygdala (BLA) to the central nucleus of the amygdala (CeA; Tye and Deisseroth, 2012; Neugebauer, 2015). This BLA-CeA circuit of glutamatergic projections has been increasingly implicated in psychiatric disorders in recent studies. For example, activation of this specific amygdala pathway induces acute anxiety behavior in mice (Tye et al., 2011). Thus, signals from BLA to CeA may rep- 
resent processed commanding information from the corticolimbic network for CeA regulation of emotion responses.

Chronic pain as a strong stressor is known to cause comorbid disorders of negative emotion including anxiety, stress, and depression in pain patients (Price, 2000; McWilliams et al., 2003; Lumley et al., 2011; Liu and Chen, 2014). The conditions of negative emotion further aggravate pain sensitivity, duration, and life experience under pain, making it a major clinical challenge at present to effectively treat chronic pain (Wilson et al., 2001; Micó et al., 2006; Wiech and Tracey, 2009; Bushnell et al., 2013). Due to the complex interactions of pain and emotion and to the lack of effective tools to separate pain and emotion processing, our understanding of the pain-emotion interactions is rather limited and related preclinical studies on underlying mechanisms in animal models are scarce.

Amygdala and especially CeA has been well established for its role in modulating the emotion dimension of pain as well as the sensory dimension of pain (Apkarian et al., 2009; Neugebauer, 2015). Through conventional immunohistochemical and pharmacological studies, it is now known that CeA receives peripheral pain signals from the glutamatergic projections of the parabrachial nucleus $(\mathrm{PBN})$ via the spino-parabrachial pathway that relays the pain signals from the spinal cord to CeA (Hunt and Mantyh, 2001; Han et al., 2015; Neugebauer, 2015; Sugimura et al., 2016). Interestingly, a recent study shows that PBN neurons containing calcitonin gene-related peptide (CGRP) and their projections to CeA relay the affective pain signals and its stimulation induces defensive responses and a threat memory (Han et al., 2015). As a major output of the amygdala complex, CeA modulates pain responses by its projections to the periaqueductal gray, a critical part of the well-established descending pain-modulating pathway in the brainstem (Fields, 2004; Basbaum et al., 2009; Neugebauer, 2015). Thus, signals from PBN to CeA convey peripheral information of pain for CeA regulation of pain-associated emotion responses.

As implicated by recent studies, CeA may play a pivotal role in integrating pain signals from the peripheral via the $\mathrm{PBN}-\mathrm{CeA}$ pathway and the modulatory signals from the corticolimbic network via the BLA-CeA pathway for regulation of emotion responses. The two excitatory glutamatergic inputs from $\mathrm{PBN}$ and BLA, and converged in CeA, could act as key players in CeA integration and regulation of emotion responses. However, the neuronal circuits that drive the pain-induced state of negative emotion are still largely unknown and how the two differential excitatory inputs in CeA interact in the context of pain-emotion interactions for behavioral outputs remains unclear. In this study, we used optogenetic stimulation to activate, in real-time, these specific excitatory projections in CeA from PBN and BLA, and identified their opposing roles in driving various behaviors of negative emotion and sensory responses.

\section{Materials and Methods}

Animals. All procedures involving the use of animals conformed to the guidelines set by the Institutional Animal Care and Use Committee of M. D. Anderson Cancer Center. Male Wistar rats (250-300 g) were used in this study. The rats were housed in groups of three with food and water available ad libitum, and in a $12 \mathrm{~h} \mathrm{light/dark} \mathrm{cycle.} \mathrm{All} \mathrm{behavioral} \mathrm{exper-}$ iments and tests were performed between 8:00 A.M. and 6:00 P.M. To induce a persistent pain condition in some experiments, a rat received a single intraplantar injection of complete Freund's adjuvant (CFA; $50 \mu \mathrm{l}$ ) in a hindpaw and experiments were conducted $3 \mathrm{~d}$ after the CFA injection.

Adeno-associated viral vectors and viral injections into PBN and BLA. Adeno-associated viral (AAV) particles of serotype 5 were obtained from the Vector Core Facility at The University of North Carolina at Chapel
Hill. An AAV5-CaMKII $\alpha$-hChR2 (H134R)-mCherry vector (AAV-ChR2) or a control vector AAV5-CaMKII $\alpha$-mCherry (AAV-mCherry) was bilaterally injected ( $1 \mu \mathrm{l}$ each side) into PBN (anteroposterior, $-9.2 \mathrm{~mm}$ from the bregma; lateral, $\pm 2.1 \mathrm{~mm}$; ventral, $-6.6 \mathrm{~mm}$ from dura), and AAV-ChR2 or a control vector AAV5-CaMKII $\alpha$-GFP (AAV-GFP) was bilaterally injected into BLA (anteroposterior, $-2.8 \mathrm{~mm}$ from the breg$\mathrm{ma}$; lateral, $\pm 5.1 \mathrm{~mm}$; ventral, $-8.2 \mathrm{~mm}$ from dura; Paxinos and Watson, 1986) in rats under anesthesia in a stereotaxic instrument. For simultaneous stimulation of both PBN-CeA and BLA-CeA projections, AAV-ChR2 or the control vector was similarly injected into the PBN and BLA of the same rat. Experiments were performed at least 4 weeks after the vector injection. After the experiments, brain tissues were harvested for anatomical identification of the injection sites. Data from injections that were outside of the targeted area were excluded.

Implantation of optical fiber cannula for optical stimulation. Three weeks after the viral injection, a mono fiberoptic cannula (Doric Lenses) was stereotaxically implanted on each side of the brain just above the CeA (anteroposterior, $-2.3 \mathrm{~mm}$ from bregma; lateral, $\pm 4.0 \mathrm{~mm}$; ventral, $-7.5 \mathrm{~mm}$ from dura) in anesthetized rats. After the implantation surgery, the animals were single housed and allowed to recover for $7 \mathrm{~d}$ before behavioral tests. For optical stimulation, the implanted cannula was connected to a $473 \mathrm{~nm}$ DPSS laser (Shanghai Laser \& Optic Century) through a fiberoptic patch cord with a rotary joint for free movement of the animal. Blue light pulses of $20 \mathrm{~Hz}, 15 \mathrm{~ms}$, and $5 \mathrm{~mW}$ with 17.68 $\mathrm{mW} / \mathrm{mm}^{2}$ or $2.5 \mathrm{~mW}$ with $8.84 \mathrm{~mW} / \mathrm{mm}^{2}$ were delivered to the CeA via the implanted cannula. Intensity of the fiberoptic light at the end of fiber was verified before and after each experiment by a power meter (PM-100D, Thor Laboratories). All laser outputs were controlled by a Master- 8 pulse stimulator (AMPI). We have shown that these stimulation parameters are effective on animal behaviors in vivo (Cai et al., 2014). CeA has been divided into lateral $\mathrm{CeA}(\mathrm{CeAl})$ and medial $\mathrm{CeA}(\mathrm{CeAm})$ with differential synapses and possibly functions (Tye et al., 2011; Neugebauer, 2015). However, in this study, we intended to stimulate all excitatory terminals in CeA from PBN or from BLA. Thus, we did not attempt to stimulate only $\mathrm{CeAl}$ or only CeAm in our experimental settings. No difference in behavioral effects was observed with different locations of the optical fiber within CeA.

Open field test. The open field test (OFT), a classical test to measure anxiety-like behaviors in rodents (Fernando and Robbins, 2011), was conducted in an illuminated chamber $(72 \times 72 \times 30 \mathrm{~cm})$ divided by a central zone and an outer zone. A rat was connected to the light source with a patch cord to the implanted optical cannula, and was placed in the center of the chamber. In a real-time OFT, a rat was allowed to move freely for $15 \mathrm{~min}$ and locomotion activity of the animal in the two zones was video-recorded and analyzed with an automated video-tracking system (EthoVision XT, Noldus Information Technology). The total test time of 15 min was divided into three consecutive 5 min periods with the light off in the first period (control). Light stimulation was given during the second 5 min period and light was off again in the third period. Reduced time spent in the unprotected central zone (central time) was regarded as anxiety-related indices. The total distance traveled in the entire chamber during the test was recorded and used as a measure of general locomotor activity.

Forced swim test. The forced swim test (FST), a main behavioral test for depression-like behaviors in rodents (Fernando and Robbins, 2011), was conducted in a cylinder (diameter $30.5 \times$ height $45.7 \mathrm{~cm}$ ) for rats $(\mathrm{ENV}$ 590R, Med Associates) according to the protocol described by Slattery and Cryan (2012). On day 1, a rat was placed into the water-filled cylinder for $15 \mathrm{~min}$ pretest swim. On day 2, the rat was connected with a patch cord to the implanted cannula and was allowed to swim for $5 \mathrm{~min}$. Light was delivered $1 \mathrm{~min}$ before and during the $5 \mathrm{~min}$ swim test. The swim activity was videotaped and immobility time was counted manually. Immobility was defined as cessation of active swimming and escaping activities. Time the animal spent immobile during the test was recorded as a measure of despair-like behavior.

Test of conditioned place preference and conditioned place aversion. Detailed procedures of conditioned place preference (CPP) and conditioned place aversion (CPA) have been described in our previous studies (Zhu et al., 2007; Bie et al., 2009; Cai et al., 2013). CPP and CPA tests were 
conducted in a standard three-chamber CPP apparatus (MED Associates). After habituation to the test chambers, a rat was placed in the center chamber and was allowed to move freely among the chambers for $15 \mathrm{~min}$ in a pretest. The time the rat spent in each chamber was recorded automatically. Then, the rat received four conditioning sessions for $8 \mathrm{~d}$, each session consisting of light stimulation-pairing conditioning for $30 \mathrm{~min}$ on one day and no light stimulation-pairing conditioning for $30 \mathrm{~min}$ on the following day. After the four conditioning sessions, the rat underwent a post-test for $15 \mathrm{~min}$ with the same procedures of the pretest. The $\mathrm{CPP} / \mathrm{CPA}$ score was defined as the difference in time the rat spent in the light-paired chamber between the pretest before conditioning treatment and the post-test in the same rat.

Analgesia test for thresholds of thermal pain. A rat was placed in a Plantar Test Instrument (Model 37370, Ugo Basile). Paw withdrawal response to an infrared heat stimulus was measured with a Hargreaves apparatus. Latency from the onset of the heat stimulus to the paw withdrawal was recorded automatically by the apparatus as threshold and was measured twice with a $5 \mathrm{~min}$ interval. The thresholds were measured before optical stimulation as baseline control and $50 \mathrm{~min}$ after $5 \mathrm{~min}$ optical stimulation. The data presented were the averaged values of paw withdrawal thresholds of both right and left hindpaw measured alternatively.

von Frey test for thresholds of mechanical pain. A rat was extensively handled and habituated to the test environment and test apparatus for $3 \mathrm{~d}$ before the pain test. Then, the rat was placed in a plastic box with mesh floor and allowed to acclimate for $20 \mathrm{~min}$. A series of calibrated von Frey filaments were applied perpendicularly to the plantar surface of a hindpaw with sufficient force to bend the filament for $6 \mathrm{~s}$. A brisk movement of the hindpaw (withdrawal or flinching) was considered as a positive response. The threshold (g) of the tactile stimulus producing a $50 \%$ likelihood of withdrawal was determined by the "up-down" calculating method (Zhang et al., 2014). The threshold was measured before optical stimulation as baseline control and after $5 \mathrm{~min}$ optical stimulation. The hindpaw withdrawal response was measured twice with a $5 \mathrm{~min}$ interval.

All behavioral tests of emotion and pain described above have been reported in our previous studies (Zhu et al., 2007; Zhang et al., 2011, 2014; Cai et al., 2013; Hou et al., 2015).

Immunohistochemistry. A rat was deeply anesthetized with pentobarbital and transcardially perfused with heparinized saline and subsequently with ice-cold $4 \%$ paraformaldehyde in $1 \times \mathrm{PBS}, \mathrm{pH}$ 7.4. The brain was removed and postfixed in $4 \%$ paraformaldehyde overnight at $4^{\circ} \mathrm{C}$, followed by dehydration with $30 \%$ sucrose in $1 \times$ PBS. Tissues were sectioned into $30-\mu \mathrm{m}$-thick coronal sections with a cryostat at $-20^{\circ} \mathrm{C}$. Sections were blocked with 5\% normal donkey serum in PBS containing $0.3 \%$ Triton X-100 and incubated overnight with primary antibodies: mouse or rabbit anti-mCherry antibody, 1:500 dilution (Abcam, Catalog \#ab167453; RRID:AB_2571870; or Abcam, Catalog \#ab125096; RRID: AB_11133266), rabbit anti-c-fos antibody, 1:200 (Santa Cruz Biotechnology, Catalog \#sc-52; RRID:AB_2106783), or anti-GFP antibody, 1:1000 (Millipore, Catalog \#MAB3580; RRID:AB_94936; or Invitrogen, Catalog \#A-11122; RRID:AB_221569). Sections were then rinsed and incubated with the goat anti-rabbit or goat anti-mouse AlexaFluorconjugated secondary antibody, 1:500 (Invitrogen, Catalog \#A-11008; RRID:AB_143165; or Catalog \#A-11004; RRID:AB_141371), and were mounted on slides, dried, and coverslipped with ProLong Gold anti-fade reagent for staining with the fluorescent reporter 4',6-diamidino-2phenylindole (DAPI; Invitrogen). The stained sections were examined with an Olympus BX51 fluorescence microscope or a Zeiss 710 confocal microscope. Intensity of fluorescence signals for c-fos staining in images was automatically quantified and analyzed by HCImage software (Hamamatsu). For each group, 12 slices from four different mice were selected. The number of c-fos-positive cells and DAPI-positive cells were counted manually by experimenters blind to experimental conditions in randomly selected, mCherry-positive areas within CeA in the images. Cells with immunofluorescence intensity $>2$-fold that of background immunofluorescence intensity were considered as c-fos-positive cells.

Statistical analysis. Comparisons of averages of two groups were performed with the two-tailed, unpaired Student's $t$ test. Two-way ANOVA for repeated measures with post hoc analysis of the Bonferroni method was used to determine statistical significance in behavioral experiments for effects of treatment and between-group interactions at each time point. A $p$ value $<0.05$ was considered statistically significant. All statistical analyses were performed with the Prism v6.0 (GraphPad). Data are presented as mean \pm SEM.

\section{Results}

Excitatory projections from PBN and BLA to CeA

We used a viral vector and immunomicroscopy to identify axon projections of excitatory neurons in the PBN and in the BLA to the CeA in rats. The AAV-ChR2 vector or AAV-mCherry control vector was bilaterally infused into the PBN of naive rats (Fig. $1 A$ ) to transfect local excitatory (CaMKII $\alpha$-expressing) neurons and their axon terminals in their projection areas. For BLA-CeA projections, AAV-ChR2 or AAV-GFP control vector was similarly infused into BLA. Four weeks later, we examined mCherry and GFP expression in PBN and in amygdala. Intense mCherry staining was observed in PBN, suggesting successful transfection of local excitatory neurons by the vector through CaMKII $\alpha$ promoters (Fig. 1B). In amygdala, we found robust mCherry expression in CeA, but not in the neighboring BLA (Fig. $1 C, D$ ). Intense GFP staining was found in BLA for successful transfection of excitatory BLA neurons (Fig. 1E). Abundant GFP staining was observed in CeA, particularly lateral CeA, demonstrating BLA-CeA projections (Fig. 1E,F). These results illustrate strong, target-specific excitatory projection from $\mathrm{PBN}$ to $\mathrm{CeA}$, a brain circuit that conveys nociceptive signal, and excitatory projection from BLA to CeA, a brain circuit that carries modulatory signal from cortical structures through their reciprocal connections with BLA.

\section{Stimulation of $\mathrm{PBN}-\mathrm{CeA}$ projection induces anxiety- and depression-like behaviors}

Because clinical studies clearly show that chronic pain conditions in patients often lead to disorders of negative emotion, such as anxiety and depression (Price, 2000; Lumley et al., 2011; Liu and Chen, 2014), we were wondering whether activating the PBNCeA projection to simulate pain signals but in an acute setting in pain-free animals would cause changes in emotion behaviors. First, we examined anxiety-like behavior by the OFT in naive rats injected bilaterally with AAV-ChR2 or AAV-mCherry into PBN. The PBN-CeA projection was optically stimulated in real-time by bilaterally implanted optical fibers in CeA. We found that, four weeks after the vector injection, AAV-ChR2- and control vector-injected rats displayed a similar level of anxiety-like behavior (time spent in central zone or central time: control: $24.1 \pm 1.9 \mathrm{~s}$, ChR2: $26.3 \pm 4.1 \mathrm{~s}, t_{(10)}=0.55, p=0.595$; distance traveled in central zone or central distance: control: $4.35 \pm 0.48 \mathrm{~m}, \mathrm{ChR} 2$ : $\left.4.25 \pm 0.65 \mathrm{~m}, t_{(10)}=0.132, p=0.898\right)$ during the initial $5 \mathrm{~min}$ period without stimulation (light off) in the $15 \mathrm{~min}$ OFT. However, after optical stimulation (light on) in CeA in the following 5 min period, analysis of two-way ANOVA revealed a significant main difference between the control vector- and the AAV-ChR2injected animal groups (group interaction: $F_{(2,20)}=6.44, p=$ 0.0069). The AAV-ChR2-injected rats showed dramatically increased anxiety behavior compared with the control rats (central time: control: $34.0 \pm 6.5 \mathrm{~s}$, ChR2: $10.8 \pm 4.1 \mathrm{~s}, t_{(10)}=2.70, p=$ 0.022; central distance: control: $4.20 \pm 0.61 \mathrm{~m}$, ChR2: $1.92 \pm$ $0.51 \mathrm{~m}, t_{(10)}=2.70, p=0.022$; Fig. $\left.2 A-C\right)$. During the immediately next $5 \mathrm{~min}$ period without stimulation (light off), the behavior of increased anxiety remained significant in ChR2-injected animals (central time: control: $19.0 \pm 4.1 \mathrm{~s}, \mathrm{ChR} 2: 6.6 \pm 2.2 \mathrm{~s}, t_{(10)}=2.34$, $p=0.041$; central distance: control: $2.59 \pm 0.22 \mathrm{~m}$, ChR2: $1.06 \pm$ $\left.0.32 \mathrm{~m}, t_{(10)}=4.10, p=0.002\right)$, which suggests that the increase in anxiety behavior is not transient. In contrast, the total distance 

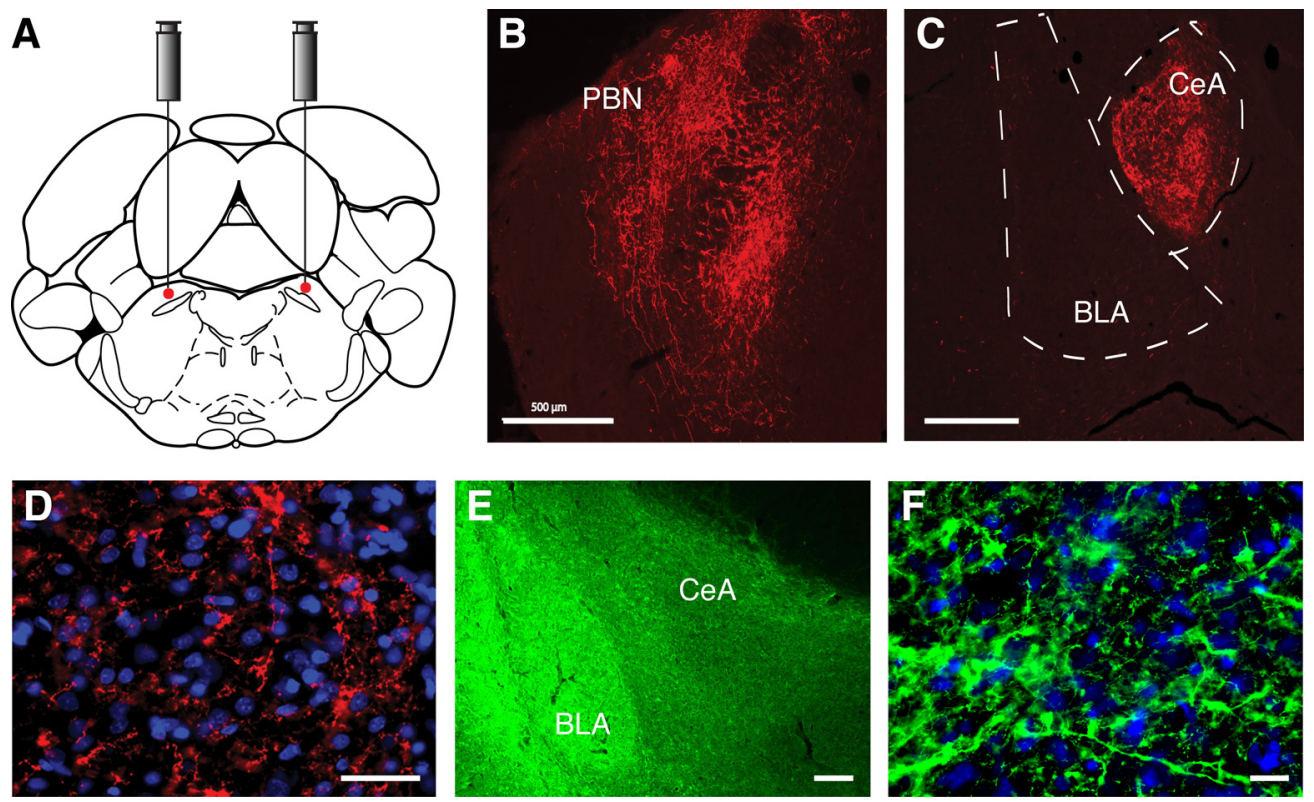

Figure 1. Excitatory PBN and BLA projections to CeA. $A, A$ diagram illustrating bilateral delivery of a viral vector into the PBN in rats. Red dots indicate PBN. $B, C$, Representative immunohistochemical images of ChR2-mCherry expression in PBN (B) and in the CeA (C), but not in the BLA, $10 \mathrm{~d}$ after bilateral infusion of the viral vector AAV5-CaMKIl $\alpha$-ChR2-mCherry into PBN of a rat. $\boldsymbol{D}$, CeA image in higher-magnification for mCherry-expressing terminals (red) and DAPI-stained cells (blue). $E$, Image of ChR2-GFP expression in BLA and CeA $10 \mathrm{~d}$ after bilateral infusion of the viral vector AAV5-CaMKIl $\alpha$-ChR2-GFP into BLA of a rat. $\boldsymbol{F}$, CeA image in higher-magnification for GFP-expressing terminals (green) and DAPI-stained cells (blue). Scale bars: $\boldsymbol{B}, \boldsymbol{C}, 500 \mu \mathrm{m} ; \boldsymbol{D}, 100 \mu \mathrm{m} ; \boldsymbol{E}, 200 \mu \mathrm{m}$; and $\boldsymbol{F}, 20 \mu \mathrm{m}$.
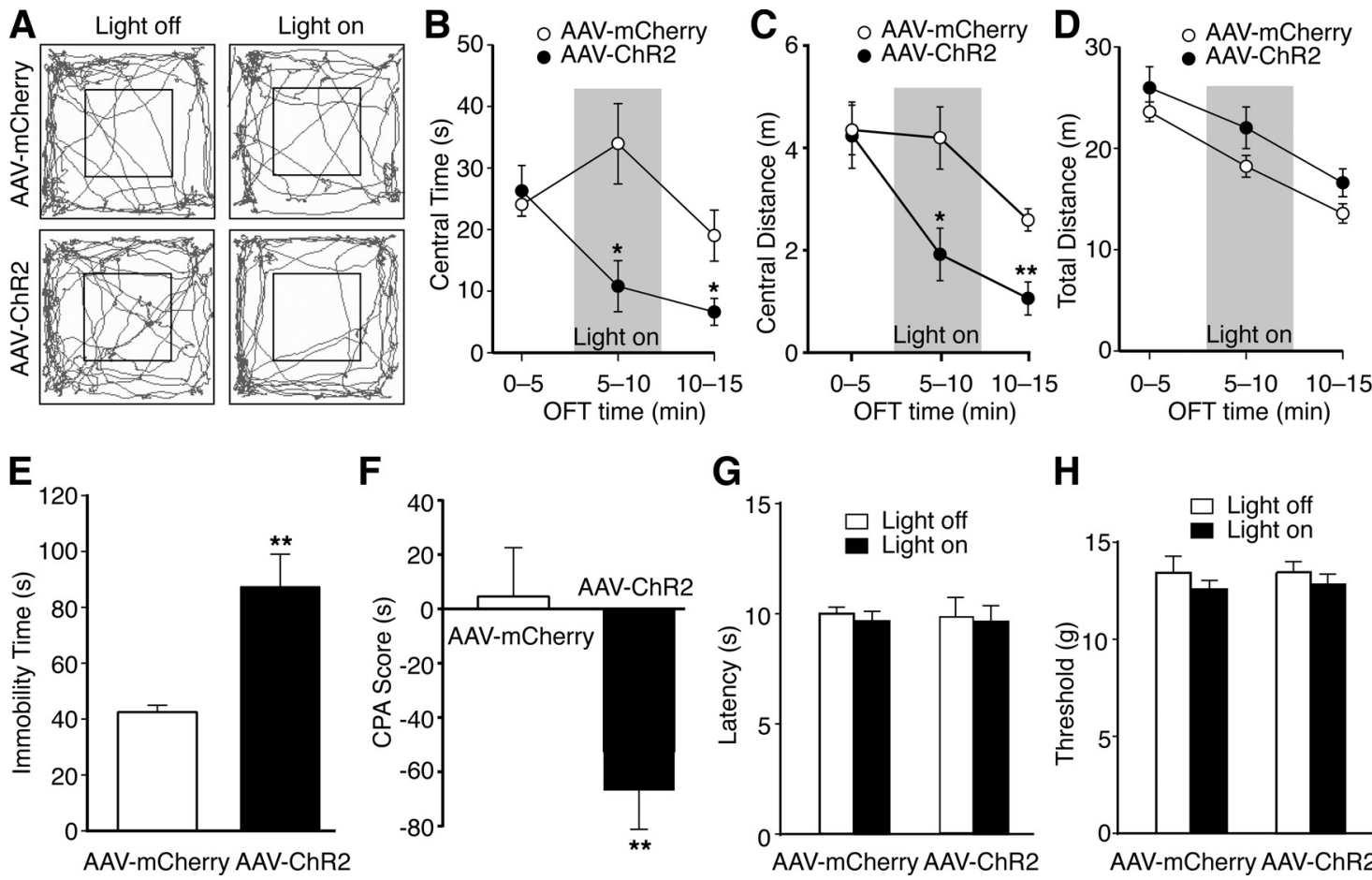

$\mathbf{F}$

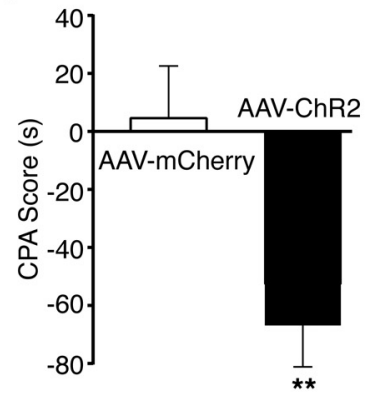

G

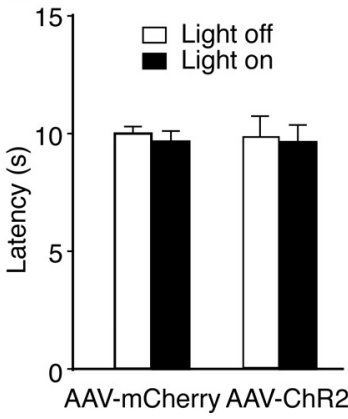

$\mathbf{H}$

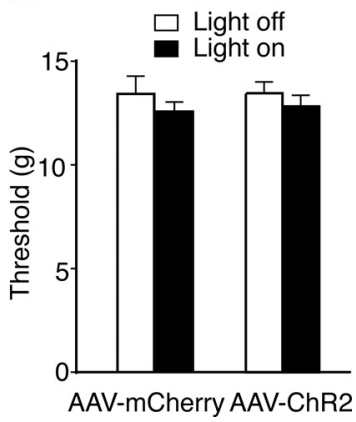

Figure 2. Optogenetic stimulation of the PBN-CeA projection in CeA induces behaviors of negative emotion. $A$, Locomotion traces of rats with PBN infusion of a control vector AAV-mCherry and the AAV-ChR2 vector for real-time optical stimulation in CeA during light-off and light-on periods in the OFT. $\boldsymbol{B}-\boldsymbol{D}$, Group data of time spent $(\boldsymbol{B})$ and distance traveled $(\boldsymbol{C})$ in central zone and total distance traveled $(\boldsymbol{D})$ in rats with PBN injection of the control vector $(n=5)$ and AAV-ChR2 vector $(n=7)$ in three consecutive 5 min periods of OFT. The light was on during the second period (gray areas) for optical stimulation. $\boldsymbol{E}$, Immobility time in rats with PBN infusion of the control vector $(n=6)$ and AAV-ChR2 $(n=6)$ during optical stimulation in CeA in the FST. $\boldsymbol{F}$, Scores of CPA in rats with PBN infusion of the control vector $(n=6)$ and AAV-ChR2 $(n=6)$ after four conditioning sessions paired with the optical stimulation in CeA. $\boldsymbol{G}, \boldsymbol{H}$, Paw-withdrawal latencies for thermal pain $(\boldsymbol{G})$ and paw-withdrawal thresholds for mechanical pain $(\boldsymbol{H})$ before (light-off) and after (light-on) optical stimulation in rats with PBN infusion of the control vector $(n=6)$ and AAV-ChR2 $(n=7)$. ${ }^{*} p<0.05,{ }^{* *} p<0.01$. 
A
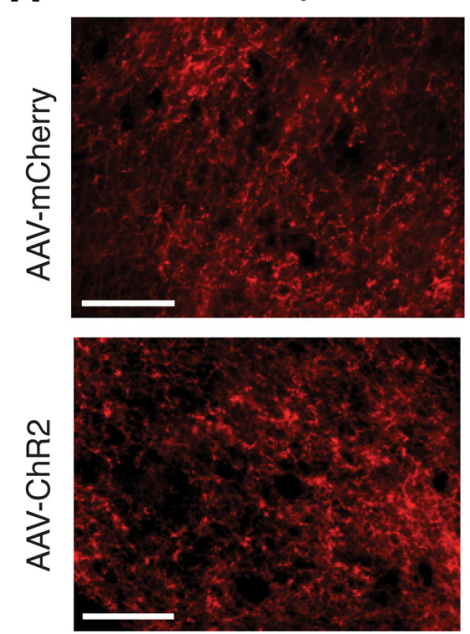
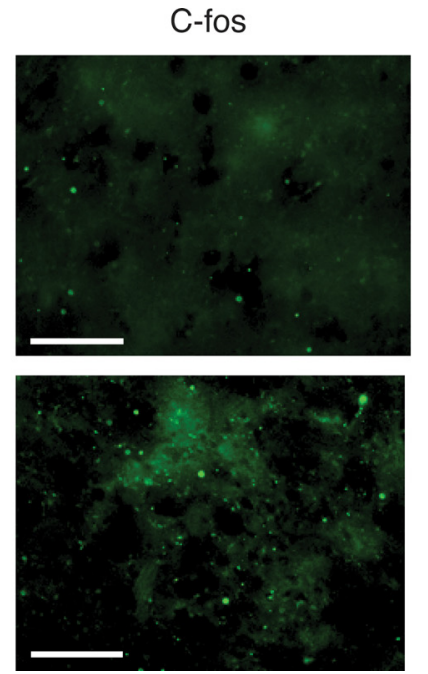
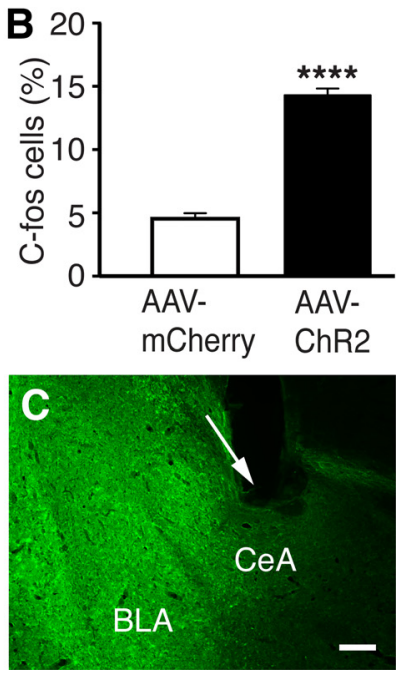

Figure 3. Optogenetic stimulation of the PBN-CeA projection in CeA increases number of c-fos-positive neurons in CeA. $\boldsymbol{A}$, Immunohistochemical images of mCherry and c-fos expression in CeA $2 \mathrm{~h}$ after $20 \mathrm{~min}$ light stimulation in rats with bilateral infusion of the control vector and AAV-ChR2 into PBN. $\boldsymbol{B}$, Summarized data of c-fos-positive neurons in randomly selected areas of CeA from the control vector-injected $(n=4)$ and AAV-ChR2-injected $(n=4)$ rats. $\boldsymbol{C}$, Image showing the tract (arrow) of a cannula fiber targeting CeA. Scale bars: $\boldsymbol{A}, 100 \mu \mathrm{m} ; \boldsymbol{C}, 200 \mu \mathrm{M}$. ${ }^{* * * *} p<0.0001$.

traveled during each of the three 5 min periods was not different between the two rat groups (interaction: $F_{(2,20)}=0.3042, p=$ 0.7411 ; light-on periods: $\left.t_{(10)}=1.79, p=0.104\right)$, and no freezing behavior was observed in the animals, indicating no impairment in the animal's locomotor activity under this stimulation protocol (Fig. 2D). These results suggest that acute activation of the excitatory PBN-CeA projection is sufficient and effective to cause anxiety-like behavior in rats under normal condition.

Next, we determined whether stimulation of the PBN-CeA projection would induce depression-like behavior measured by the FST. In the same two groups of rats, we found that optical stimulation induced significant depression-like behavior expressed as increased immobility time (control: $42.5 \pm 2.5 \mathrm{~s}$, ChR2: $87.3 \pm$ $\left.11.7 \mathrm{~s}, t_{(10)}=3.75, p=0.0038\right)$ in AAV-ChR2-injected rats (Fig. $2 E$ ). These results support the notion that acute activation of the excitatory $\mathrm{PBN}-\mathrm{CeA}$ projection is sufficient to drive behaviors of negative emotion such as anxiety and depression in rats.

\section{Stimulation of $\mathrm{PBN}-\mathrm{CeA}$ projection induces aversion}

Pain as a strongly unpleasant experience is of aversion and PBN neurons covey pain signals from the peripheral to corticolimbic structures including amygdala in the brain for cognitive evaluation and emotion processing (Hunt and Mantyh, 2001; Baliki and Apkarian, 2015; Han et al., 2015; Neugebauer, 2015). We then determined whether stimulation of the $\mathrm{PBN}-\mathrm{CeA}$ projection would mimic this aversive effect of pain, using the paradigm of CPA. In separate groups of rats after place conditioning, we found that optical stimulation of the $\mathrm{PBN}-\mathrm{CeA}$ projection induced strong place aversion in ChR2-injected rats, but not in control rats, in the CPA test (CPA score: control: $4.6 \pm 18.0 \mathrm{~s}, \mathrm{ChR} 2:-67.4 \pm 13.1 \mathrm{~s}$, $t_{(15)}=3.29, p=0.005$; Fig. $\left.2 F\right)$. Thus, it appears that stimulating the $\mathrm{PBN}-\mathrm{CeA}$ projection share another key feature of pain, inducing strong negative reinforcement as measured by place aversion in rats.

An intriguing question was whether this stimulation of the PBN-CeA projection that simulates pain signals would change behavioral response to pain itself. We then assessed pain responses of mechanical allodynia and thermal hyperalgesia in additional groups of normal rats injected with AAV-ChR2 or control vector. Surprisingly, we found that the same optical stimulation of the PBN-
CeA projection caused no change in pain behaviors of either mechanical allodynia or thermal hyperalgesia (Fig. 2G,H). This finding is in strong contrast to previous reports that hyperactivity of CeA neurons is induced by a general increase in excitatory synaptic transmission under various pain conditions both in animal models and in humans (Simons et al., 2014; Hou et al., 2015; Neugebauer, 2015).

\section{Stimulation of $\mathrm{PBN}-\mathrm{CeA}$ projection increases cell activity in $\mathrm{CeA}$}

To validate that the optical stimulation we used was effective in activating target neurons, we assessed changes in cell activity in ChR2- and control vector-injected rats, using c-fos as a marker of cell activity (Cai et al., 2014; Adhikari et al., 2015; Kim et al., 2016; Kitamura et al., 2017). As expected, our general survey of CeA c-fos imaging showed that, after the optical stimulation in CeA, the number of c-fos-positive cells was significantly increased in the CeA of ChR2-injected rats compared with that of control rats (control: $4.5 \pm 0.4 \%$, ChR2: $14.3 \pm 0.5 \%, t_{(22)}=13.9, p<$ 0.0001 ; Fig. $3 A, B$ ), confirming that the stimulation protocol to activate the excitatory projection terminals from $\mathrm{PBN}$ in $\mathrm{CeA}$ increases cell activity in CeA. Figure $3 C$ illustrates a tract of optical fiber targeting CeA for optical stimulation.

\section{Stimulation of BLA-CeA projection reduces anxiety and depression}

In the next series of experiments, we used a similar viral strategy of optical stimulation, but injected the vectors into BLA to stimulate the excitatory projection from BLA to CeA in additional groups of rats. In the OFT for anxiety behavior, the real-time optical simulation of the BLA-CeA projection in CeA clearly decreased anxiety behavior, with significantly increased central time in ChR2-injected rats during the $5 \mathrm{~min}$ light-on period (control: $24.6 \pm 0.8 \mathrm{~s}, \mathrm{ChR} 2: 29.7 \pm 1.2 \mathrm{~s}, t_{(12)}=2.62, p=0.022$; Fig. $4 A$ ). This effect was completely opposite to the effect of stimulating the PBN-CeA projection on anxiety behavior (Fig. $2 A-C$ ). This behavior of reduced anxiety remained significant during the following 5 min light-off period with no stimulation (control: $17.7 \pm 0.8 \mathrm{~s}$, ChR2: $\left.22.6 \pm 1.6 \mathrm{~s}, t_{(12)}=3.16, p=0.008\right)$. The total distance traveled was unchanged in the ChR2 group (group in- 

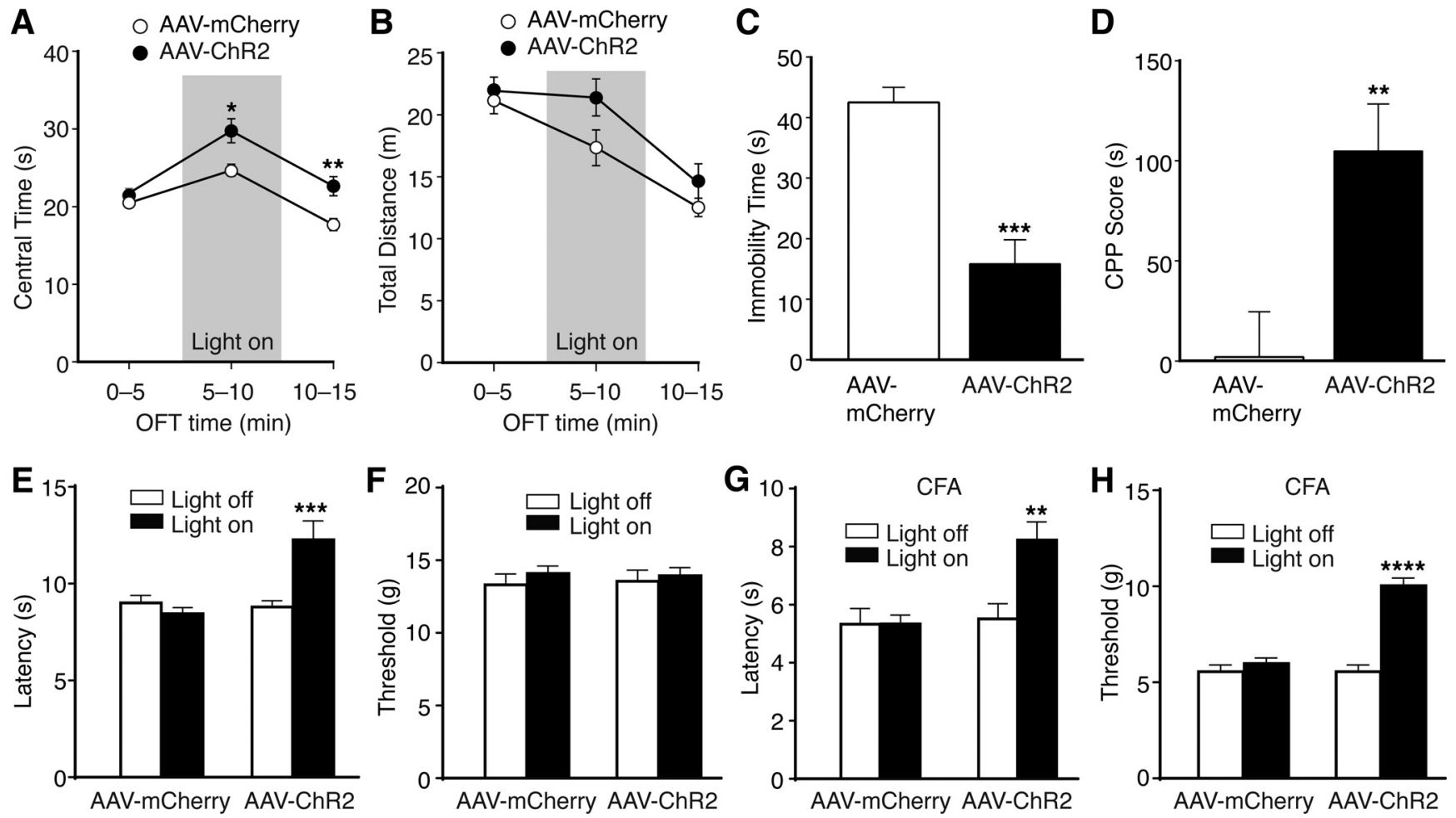

Figure 4. Optogenetic stimulation of the BLA-CeA projection in CeA inhibits behaviors of negative emotion and sensory pain. $A, B$, Group data of time spent in central zone $(\boldsymbol{A})$ and total distance traveled $(\boldsymbol{B})$ in rats with bilateral infusions of the control vector $(n=6)$ and AAV-ChR2 $(n=8)$ into BLA in three consecutive 5 min periods of OFT. The light was on during the second period. C, Immobility time in rats with BLA infusions of the control vector $(n=6)$ and AAV-ChR2 $(n=6)$ during optical stimulation in CeA in FST. D, Scores of CPP in rats with BLA infusions of the control vector $(n=6)$ and AAV-ChR2 $(n=6)$ after four conditioning sessions paired with the optical stimulation in CeA. $\boldsymbol{E}, \boldsymbol{F}$, Paw-withdrawal latencies for thermal pain $(\boldsymbol{E})$ and paw-withdrawal thresholds for mechanical pain $(\boldsymbol{F})$ before (light-off) and after (light-on) optical stimulation in normal rats with BLA infusions of the control vector $(n=6)$ and AAV-ChR2 $(n=7)$ in rats. $\boldsymbol{G}, \boldsymbol{H}$, Results of similar behavioral tests for thermal pain $(\boldsymbol{G})$ and mechanical pain $(\boldsymbol{H})$ in the vector-injected rats (AAV-mCherry, $n=8 ; \mathrm{AAV}-\mathrm{ChR2}, n=6) 3 \mathrm{~d}$ after an intraplantar injection of CFA. ${ }^{*} p<0.05$, ${ }^{* *} p<0.01$, ${ }^{* * *} p<0.001,{ }^{* * * *} p<0.0001$.

teraction: $F_{(2,24)}=1.3, p=0.2909$; light-on periods: control: $17.4 \pm 1.2 \mathrm{~m}$, ChR2: $21.4 \pm 1.5 \mathrm{~m}, t_{(12)}=1.99, p=0.070$; Fig. $4 B)$.

Next in the FST, we determined the behavioral effect of stimulating the BLA-CeA projection on depression-like behavior in the same two groups of rats. Consistent with its effect on anxiety, optically stimulating the BLA-CeA projection decreased depression-like behavior with significantly decreased immobility time in the ChR2 group (control: $42.5 \pm 2.5 \mathrm{~s}$, ChR2, $15.8 \pm 4.0 \mathrm{~s}, t_{(10)}=$ $5.68, p=0.0002$; Fig. $4 C$ ), an effect also opposite to that of stimulating the $\mathrm{PBN}-\mathrm{CeA}$ projection (Fig. $2 E$ ).

\section{Stimulation of BLA-CeA projection induces reward-like effect}

As pain causes aversive and unpleasant experience, pain inhibition is rewarding by inducing negative reinforcement (Fields, 2004). Because we found that stimulating the BLA-CeA projection counteracted the behaviors of negative emotion that are induced by pain conditions, we were wondering what would happen to reward-related behavior after this optical stimulation. Quite interestingly, using the paradigm of CPP in additional rats, we found that stimulating the BLA-CeA projection produced strong CPP in the ChR2 group while no change was observed in preference behavior in the control group (CPP score: control: $1.9 \pm 22.7$ s, ChR2: $105 \pm 23.6 s, t_{(15)}=3.13, p=0.0069$; Fig. $4 D)$. This is again opposite to the effect of stimulating the PBN$\mathrm{CeA}$ projection on the reward- and aversion-related preference behavior (Fig. $2 F$ ), suggesting that activation of this BLA-CeA pathway induces a reward-like behavior of positive reinforcement.

\section{Stimulation of BLA-CeA projection inhibits pain}

We then determined how stimulating the BLA-CeA projection would affect pain behavior. In additional normal rats injected with ChR2 vector or AAV-mCherry vector in BLA, we found that optical stimulation in CeA significantly inhibited thermal pain in the ChR2 group, but not in the control AAV-mCherry group, when the pain thresholds were compared before and after the optical stimulation (control: $t_{(14)}=1.03, p=0.321$; ChR2: $t_{(10)}=$ $6.08, p=0.0001$; Fig. $4 E$ ). However, mechanical pain threshold was unchanged by the stimulation in either rat group (control: $t_{(14)}=1.52, p=0.151$; ChR2: $t_{(10)}=0.25, p=0.807$; Fig. $4 F$ ). These differential effects on thermal and mechanical pain thresholds may reflect the different features of the two pain tests, as the thermal pain test measures response to a noxious (painful) stimulus while the mechanical test measures response to a non-noxious stimulus. To further assess the role of the BLA-CeA pathway in modulation of pain behaviors, we induced an inflammatory pain condition by an intraplantar injection of CFA in rats with similar infusions of the vectors into BLA. Three days after the CFA injection, the baseline thresholds of both thermal and mechanical pain responses were, as expected, significantly decreased (thermal: control: $8.9 \pm 0.3 \mathrm{~s}$, CFA: $5.3 \pm 0.5 \mathrm{~s}, t_{(14)}=5.62, p<0.0001$; mechanical: control: $13.5 \pm 0.4 \mathrm{~s}, \mathrm{CFA}: 5.6 \pm 0.4 \mathrm{~s}, t_{(14)}=15.0$, $p<0.0001$; Fig. 4G,H), indicating hyperalgesia and allodynia, respectively. Interestingly, in these rats with CFA-induced pain hypersensitivity, optical stimulation of the BLA-CeA projection significantly reduced both thermal and mechanical pain responses with increased pain threshold in the ChR2 group, but not in the control group (thermal: mCherry: $t_{(14)}=0.25, p=0.8078$, ChR2: 
A
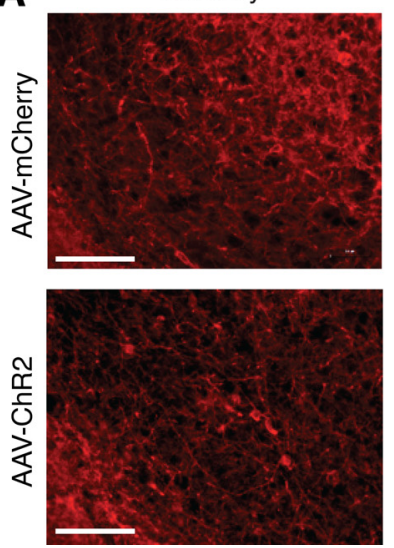
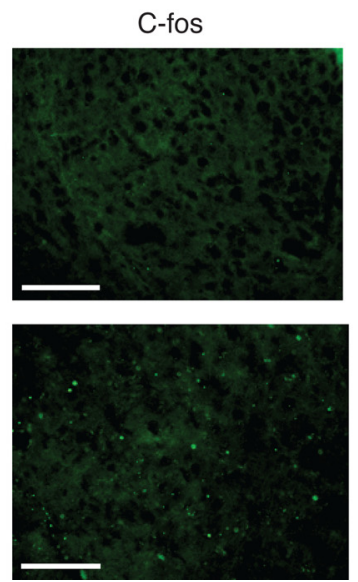

B

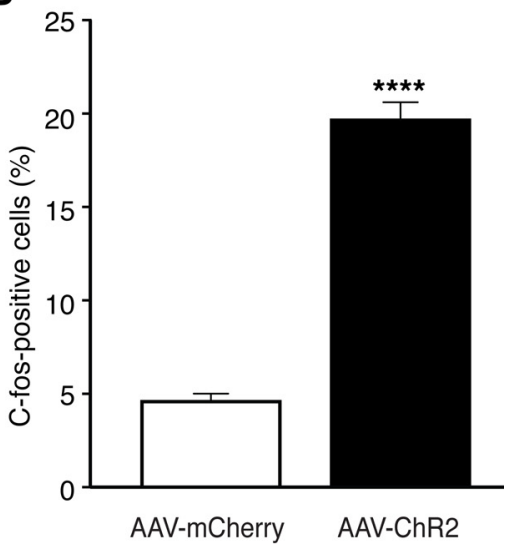

Figure 5. Optogenetic stimulation of the BLA-CeA projection in CeA increases number of c-fos-positive neurons in CeA. $A$, immunohistochemical images of mCherry and c-fos expression in CeA $2 \mathrm{~h}$ after $20 \mathrm{~min}$ light stimulation in rats $30 \mathrm{~d}$ after bilateral infusion of the control vector and AAV-ChR2 into BLA. $\boldsymbol{B}$, Summarized data of c-fos-positive neurons in randomly selected areas of CeA from the control vector-injected $(n=4)$ and AAV-ChR2-injected $(n=4)$ rats. Scale bars, $100 \mu \mathrm{m}$. ${ }^{* * * *} p<0.0001$.

$t_{(10)}=4.18, p=0.0019 ;$ mechanical: mCherry: $t_{(14)}=0.01, p>$ 0.999, ChR2: $t_{(10)}=8.22, p<0.0001$; Fig. $\left.4 G, H\right)$. These findings suggest that activation of this BLA-CeA pathway inhibits pain under normal and pain conditions.

We also examined the effect of stimulating the BLA-CeA pathway on cell activity in CeA with c-fos imaging. As shown in Figure 5, optical stimulation of this BLA-CeA projection in CeA significantly increased the number of c-fos-positive cells in CeA only in ChR2-injected rats, indicating increased cell activity in CeA after the stimulation (control: $4.6 \pm 0.4 \%$, ChR2: $19.6 \pm$ $\left.1.0 \%, t_{(22)}=14.3, p<0.0001\right)$.

\section{Simultaneous stimulation of PBN-CeA and BLA-CeA} projections fails to induce anxiety or depression

To further verify whether activation of the BLA-CeA projection indeed inhibits PBN-CeA stimulation-induced negative emotion, we determined the behavioral effects of simultaneous stimulation of both PBN-CeA and BLA-CeA projections in additional groups of rats. AAV-ChR2 or control vector (AAV-mCherry for PBN and AAV-GFP for BLA) was bilaterally infused into both the PBN and BLA of the same rat. Interestingly, we found that the same optical stimulation in the CeA of these rats failed to induce significant anxiety (central time: control: $31.8 \pm 2.2 \mathrm{~s}$, ChR2: $35.1 \pm 3.6 \mathrm{~s}$, $t_{(13)}=0.682, p=0.507$ ) or depression (immobility time: control: $36.2 \pm 6.8 \mathrm{~s}$, ChR2: $39.0 \pm 7.0 \mathrm{~s}, t_{(10)}=0.28, p=0.783$; Fig. $6 A, C)$. This is in strong contrast to the significant effects by stimulating the PBN-CeA projection alone (Fig. 4A,C). No change was observed in locomotor activity either (light-on periods: $t_{(13)}=$ $0.934, p=0.367$; Fig. $6 B$ ). However, this simultaneous stimulation of both projections still induced an inhibition on thermal pain sensitivity (control: $t_{(10)}=0.90, p=0.391$, ChR2: $t_{(14)}=$ 6.34, $p<0.0001$; Fig. $6 D$ ), as did BLA-CeA stimulation alone (Fig. $4 E$ ). These finding indicate that activation of the BLA-CeA projection inhibits the emotion effects of the $\mathrm{PBN}-\mathrm{CeA}$ projection, whereas the pain-inhibiting effect of the BLA-CeA projection is not affected by activities of the $\mathrm{PBN}-\mathrm{CeA}$ projection.

\section{Differential distributions of excitatory terminals in CeA from PBN and BLA}

Finally, we examined the anatomic distribution of terminals in CeA from the $\mathrm{PBN}-\mathrm{CeA}$ projection and from the BLA-CeA projection with confocal imaging by double injections of AAV5-CaMKII $\alpha$-ChR2mCherry into PBN and AAV5-CaMKII $\alpha$-ChR2-GFP into BLA in the same rat. As shown in Figure $7 A$, the excitatory cells in $\mathrm{PBN}$ projected strongly and specifically to $\mathrm{CeA}$, but not to BLA, despite the long distance from $\mathrm{PBN}$ to CeA. The terminals from PBN were most dense in the $\mathrm{CeAl}$ and less densely distributed in the CeAm with the least distribution in the ventromedial part of CeAm. In comparison, the excitatory terminals from BLA were relatively moderate given the short distance from BLA to CeA (Fig. $7 B$ ) and were mostly distributed in the lateral-ventral and ventromedial part of CeA (Fig. 7C). This differential distribution was further illustrated by a representative area in the middle of CeA where terminals from both PBN and BLA were present and somewhat mixed, but more terminals from PBN in the dorsolateral part of the area and more terminals from BLA in the ventral part with completely no overlaps as expected (Fig. $7 D-F$ ).

\section{Discussion}

In this study, we have presented evidence showing that activation of the PBN-CeA pathway is sufficient to drive a series of behaviors of negative emotion without the presence of pain, whereas activation of the BLA-CeA pathway opposes each of the same behaviors of negative emotion, providing a top-down regulating mechanism for cognitive control of negative emotion.

\section{Emotion-driving signals from the $\mathrm{PBN}-\mathrm{CeA}$ pathway}

Although brain structures and circuits involved in processing and regulation of emotion and pain have been well characterized, how these brain circuits convey and process peripheral signals and make cognitive decisions on corresponding adaptive responses remains largely unclear and has just begun to be explored in recent years. For peripheral pain signals, the PBN is known to relay signals of both sensory pain and affective pain to the cortical and corticolimbic structures together with the spinothalamic pathway for central processing, as the $\mathrm{PBN}-\mathrm{CeA}$ pathway is activated in several animal models of pain (Hunt and Mantyh, 2001; Han et al., 2015; Neugebauer, 2015). However, it is unknown whether the pain signals transmitted in the PBN-CeA pathway is able to directly drive behaviors of negative emotion that is often associated with chronic pain conditions. Our results in this study demonstrate that acute activation of the excitatory $\mathrm{PBN}-\mathrm{CeA}$ pathway in normal animals is sufficient to generate a series of behaviors of negative emotion, creating a behavioral state of negative emotion that is commonly present in animals with chronic pain (Hunt and Mantyh, 2001; Baliki and Apkarian, 2015; Neugebauer, 2015; 

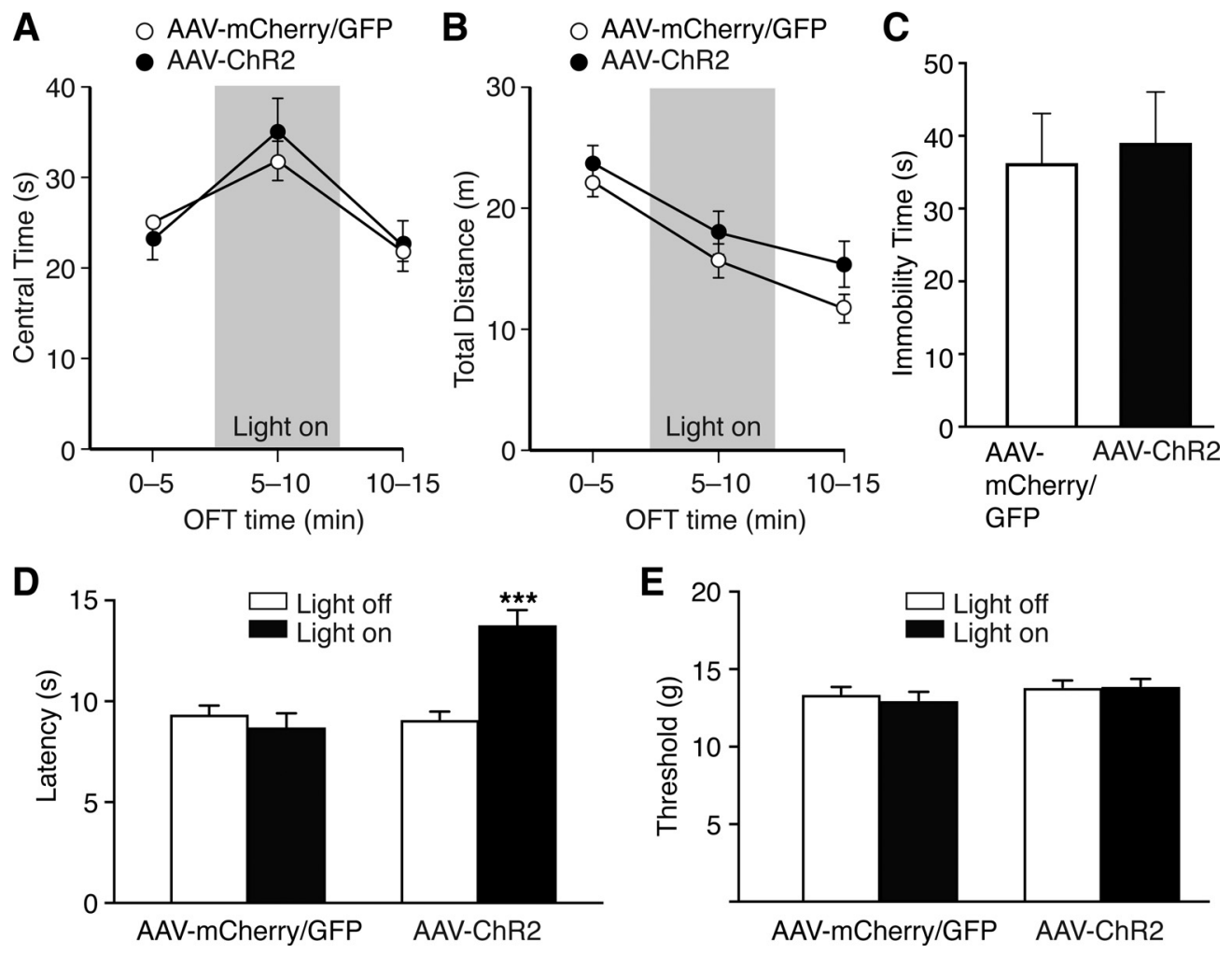

Figure 6. Simultaneous stimulation of both PBN-CeA and BLA-CeA projections in CeA does not induce behaviors of negative emotion. $\boldsymbol{A}, \boldsymbol{B}$, Group data of time spent in central zone ( $\boldsymbol{A}$ ) and total distance traveled $(B)$ of OFT in rats with bilateral infusions of the control vector $(n=6)$ and AAV-ChR2 $(n=9)$ into PBN and BLA of the same rat. The light-on period is indicated by the gray columns. C, Immobility time of FST during optical stimulation in the same rats with vector infusions into both PBN and BLA. D, E, Paw-withdrawal latencies for thermal pain (D) and paw-withdrawal thresholds for mechanical pain $(\boldsymbol{E})$ before (light-off) and after (light-on) optical stimulation in the same rats. ${ }^{* * *} p<0.001$.
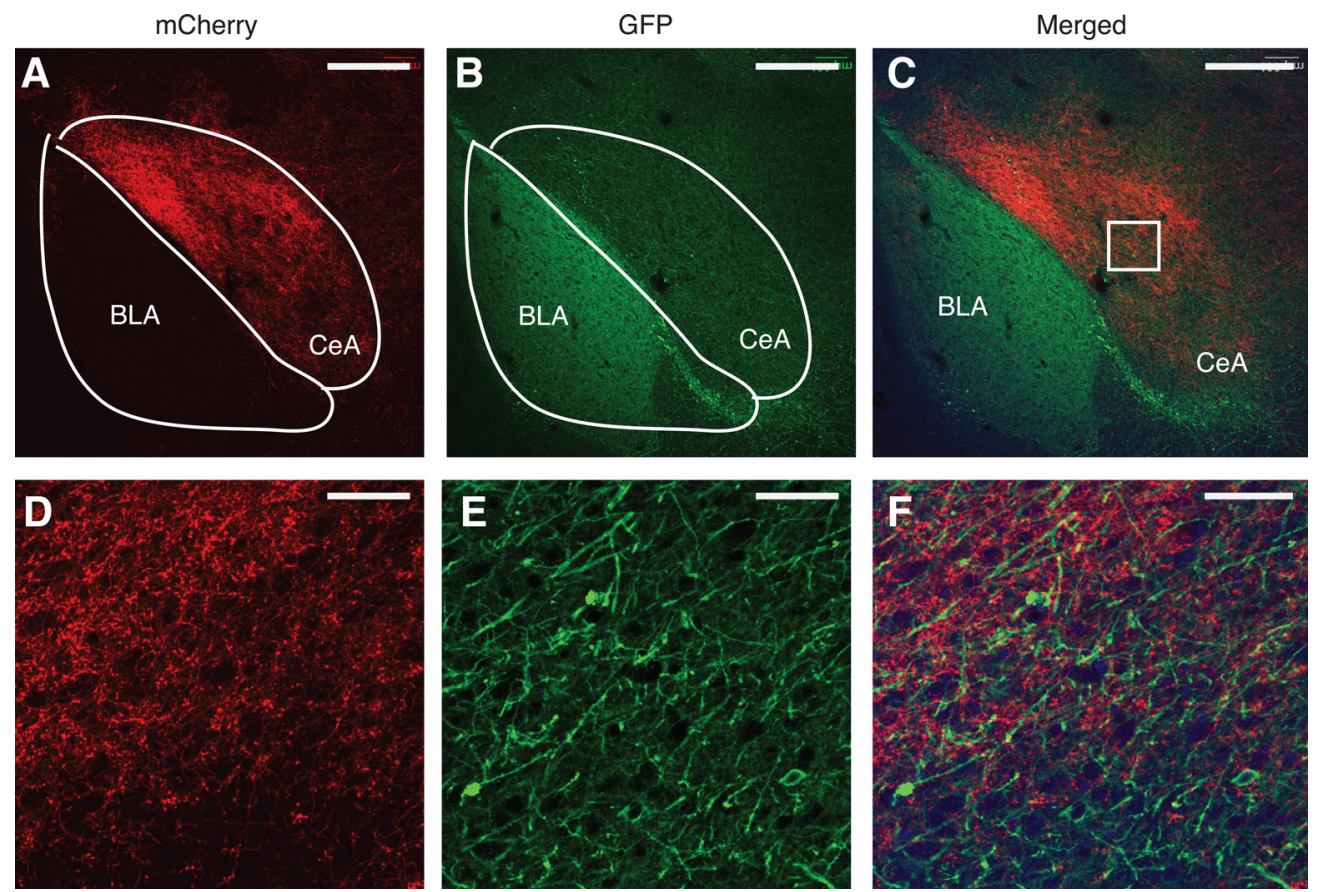

Figure 7. Differential distribution of excitatory terminals in CeA from PBN and from BLA. $\boldsymbol{A}-\boldsymbol{C}$, Representative immunohistochemical images of excitatory terminals in CeA in a rat receiving double injections of AAV-CaMKII $\alpha$-ChR2-mCherry into PBN (A, red), AAV-CaMKII $\alpha$-ChR2-GFP into BLA (B, green) in the same rat, and merged images of $\boldsymbol{A}$ and $\boldsymbol{B}(\boldsymbol{C})$. D $-\boldsymbol{F}$, CeA images of higher-magnification in the square area marked in $\boldsymbol{C}$ for PBN-CeA projection terminals $(\boldsymbol{D})$, BLA-CeA projection terminals $(\boldsymbol{E})$, and their emerged image $(\boldsymbol{F})$. Scale bars: $A-C, 250 \mu \mathrm{m} ; D, E, 50 \mu \mathrm{m}$. 
Zhuo, 2016). The excitatory PBN-CeA projection stimulated in this study is thought to be mostly glutamatergic, as they are targeted through the CaMKII $\alpha$ promoters (Tye and Deisseroth, 2012). Thus, it appears that the peripheral pain signals that reach the amygdala through the PBN-CeA pathway are capable of driving emotion behaviors, which provides a direct link between environmental stimuli and behaviors of emotional responses.

\section{Top-down regulating signals from the BLA-CeA pathway}

For emotion processing, the corticolimbic network plays a central role for evaluation and integration of emotion-related environmental inputs and adaptive behavioral responses, such as those in drug addiction and chronic pain (Deisseroth, 2014; Baliki and Apkarian, 2015; Navratilova et al., 2015). The amygdala as part of the corticolimbic circuits, particularly BLA and CeA, is important in emotional learning and behavioral integration of positive and negative emotional responses (Gottfried et al., 2003; Adhikari et al., 2015; Han et al., 2015). In some original studies of optogenetics to dissect brain circuit functions, the excitatory BLA-CeA pathway has been identified to be critical in regulating anxiety behavior. Inactivating this pathway promotes, whereas stimulating this pathway inhibits, anxiety behavior in mice, suggesting an anxiolytic role of the excitatory BLA-CeA projection (Tye et al., 2011). Our results are consistent with this anxiolytic role of the BLA-CeA projection, and significantly expand its roles to anti-depression, rewarding, and pain-inhibiting effects. Importantly, these effects directly counteract the corresponding and opposite effects induced by activation of the pain signal-transmitting PBN-CeA pathway, forming a top-down emotion control mechanism mediated by the corticolimbic circuits. Thus, we propose that, under acute to persistent pain conditions, although the pain signals conveyed to CeA may be sufficient to cause negative emotion, the corticolimbic system activated by the pain signals through spinothalamic pathways would engage this control mechanism to counteract the emotional effects of acute pain, resulting in no apparent emotional changes; however, under chronic pain conditions, the excitatory projections in the CeA circuits may undergo activity-dependent adaptations that lead to an overwhelming effect of the pain signals or an impairment in the topdown control mechanism, manifesting pain-induced behaviors of negative emotion. A recent study has demonstrated a topdown control of anxiety state and learned freezing behavior by projections from ventral medial prefrontal cortex to the basomedial amygdala (Adhikari et al., 2015). The corticolimbic process of affective pain could be highly individual-dependent, such as personal intrinsic features of the corticolimbic network, memories of life events, and prior pain experience, contributing to individual pain vulnerability to developing chronic pain (Denk et al., 2014). This top-down control mechanism and its adaptive impairment relative to the sustained emotion-driving signals could be also involved in other neuropsychiatric disorders with uncontrollable negative emotion, such as anxiety and depression, under chronic stressful conditions. Future studies are warranted to test this hypothesis.

\section{Interaction of emotion-driving signals and top-down regulating signals}

It is intriguing to mull over how the amygdala circuits and even those within CeA can mediate behavioral responses of both negative (fear, pain) and positive (reward) emotions. Are they mediated by different or the same populations of neurons and synaptic circuits? Recent studies in BLA and CeA suggest circuit-specific and neuron-specific encoding of negative and positive behavioral outcomes (Janak and Tye, 2015). The opposite behavioral outcomes of activating the two excitatory inputs to CeA neurons from $\mathrm{PBN}$ and BLA indicate two functionally distinct populations of CeA neurons, supporting the notion that local wiring of the neuronal circuits, in this case the source of inputs rather than the neurochemical (glutamatergic) property of inputs, encodes behavioral outcomes of negative or positive emotions. Our analysis of immunohistochemical imaging shows that these two types of projecting terminals are non-overlapping and differentially concentrated in the subregions of CeA, with $\mathrm{PBN}-\mathrm{CeA}$ terminals most dense in the dorsal part of CeAl and BLA-CeA terminals more abundant in the ventral part of $\mathrm{CeAl}$ and $\mathrm{CeAm}$. The CeAl has been considered as nociceptive $\mathrm{CeA}$ as it has been shown to be mostly involved in pain inputs and pain responses (Neugebauer, 2015). Given the distinct functions of these two types of glutamatergic terminals in CeA from PBN and BLA, there are likely functionally distinct or even opposing subpopulations of neurons that can be partially defined by these glutamatergic inputs within the synaptic circuits of CeA.

How the two populations of CeA neurons encoding negative and positive behavioral outcomes interact or counteract each other remains unclear. The distinct functions are likely defined by the synaptic wiring of CeA neurons. As described in previous studies on a simplified signal flow in amygdala, glutamatergic BLA neurons project to GABAergic CeAl neurons, which project to GABAergic CeAm neurons, forming a feedforward GABA inhibition of CeA outputs from CeAm (Tye et al., 2011; Neugebauer, 2015). In this model of serial circuitry, activation of glutamatergic terminals from BLA excites CeAl neurons, which then inhibit CeAm neurons via GABA, producing an anxiolytic effect, as shown in this study and previous reports (Gilpin et al., 2015; Janak and Tye, 2015). Apparently, this simplified serial model does not include functionally distinct populations of CeA neurons and synaptic wiring. Our findings that activation of glutamatergic terminals from $\mathrm{PBN}$ to $\mathrm{CeAl}$ causes an opposite anxiogenic effect suggest that (1) there are functionally distinct populations of neurons within $\mathrm{CeAl}$ and (2) there exist parallel circuits within $\mathrm{CeAl}$ that exert differential effects on CeAm output neurons or on other populations of $\mathrm{CeAl}$ neurons for distinct behavioral outcomes.

Further evidence supporting the notion of parallel CeA circuits comes from our findings that activating the $\mathrm{PBN}-\mathrm{CeA}$ projection can no longer induce negative emotions when the BLA-CeA projection is also activated at the same time. This suggests a direct inhibitory interaction between the two populations of neurons and related synaptic circuits in CeAl. It indicates that corticolimbic inputs likely have a direct inhibitory control on paindriven emotional responses through synaptic circuits in $\mathrm{CeAl}$ or via their common downstream targets. In contrast, our results of pain modulation indicate yet another feature of the CeA circuits. As activating the $\mathrm{PBN}-\mathrm{CeA}$ projection failed to alter baseline pain response or pain-inhibiting effect of the $\mathrm{BLA}-\mathrm{CeAl}$ projection, it may suggest that the $\mathrm{CeAl}$ circuit that receives BLA and corticolimbic inputs connects to the descending pain-modulating pathway in the brainstem, but not the CeAl circuit that receives nociceptive PBN inputs. It is worth noting that the optical stimulation used in the current study did not intend to target the terminals in CeAl only or CeAm only, and other neuron types and synaptic connections, including interneurons within CeAl, intercalated GABAergic cells between BLA and CeA, and BLA projections to CeAm, could all play a role in the synaptic interactions within the amygdala's BLA-CeA circuitry. 
In summary, the current study identifies that the pain signals conveyed through the PBN-CeA pathway are sufficient to drive several behaviors of negative emotion seen under chronic pain conditions and that processed signals from the BLA-CeA pathway oppose each of the same behaviors of negative emotion. This possible top-down control mechanism for negative emotion from the corticolimbic system and its potential impaired effect overwhelmed by sustained pain signals on an individual base may play a critical role in the development of comorbid disorders of negative emotion under chronic pain conditions and in the development of neuropsychiatric diseases in general.

\section{References}

Adhikari A, Lerner TN, Finkelstein J, Pak S, Jennings JH, Davidson TJ, Ferenczi E, Gunaydin LA, Mirzabekov JJ, Ye L, Kim SY, Lei A, Deisseroth K (2015) Basomedial amygdala mediates top-down control of anxiety and fear. Nature 527:179-185. CrossRef Medline

Apkarian AV, Baliki MN, Geha PY (2009) Towards a theory of chronic pain. Prog Neurobiol 87:81-97. CrossRef Medline

Baliki MN, Apkarian AV (2015) Nociception, pain, negative moods, and behavior selection. Neuron 87:474-491. CrossRef Medline

Basbaum AI, Bautista DM, Scherrer G, Julius D (2009) Cellular and molecular mechanisms of pain. Cell 139:267-284. CrossRef Medline

Baxter MG, Murray EA (2002) The amygdala and reward. Nat Rev Neurosci 3:563-573. CrossRef Medline

Bie B, Zhu W, Pan ZZ (2009) Rewarding morphine-induced synaptic function of delta-opioid receptors on central glutamate synapses. J Pharmacol Exp Ther 329:290-296.

Bushnell MC, Ceko M, Low LA (2013) Cognitive and emotional control of pain and its disruption in chronic pain. Nat Rev Neurosci 14:502-511. CrossRef Medline

Cai YQ, Wang W, Hou YY, Zhang Z, Xie J, Pan ZZ (2013) Central amygdala GluA1 facilitates associative learning of opioid reward. J Neurosci 33: 1577-1588. CrossRef Medline

Cai YQ, Wang W, Hou YY, Pan ZZ (2014) Optogenetic activation of brainstem serotonergic neurons induces persistent pain sensitization. Mol Pain 10:70. CrossRef Medline

Deisseroth K (2014) Circuit dynamics of adaptive and maladaptive behaviour. Nature 505:309-317. CrossRef Medline

Denk F, McMahon SB, Tracey I (2014) Pain vulnerability: a neurobiological perspective. Nat Neurosci 17:192-200. CrossRef Medline

Fernando AB, Robbins TW (2011) Animal models of neuropsychiatric disorders. Annu Rev Clin Psychol 7:39-61. CrossRef Medline

Fields H (2004) State-dependent opioid control of pain. Nat Rev Neurosci 5:565-575. CrossRef Medline

Gilpin NW, Herman MA, Roberto M (2015) The central amygdala as an integrative hub for anxiety and alcohol use disorders. Biol Psychiatry 77:859-869. CrossRef Medline

Gottfried JA, O’Doherty J, Dolan RJ (2003) Encoding predictive reward value in human amygdala and orbitofrontal cortex. Science 301:11041107. CrossRef Medline

Han S, Soleiman MT, Soden ME, Zweifel LS, Palmiter RD (2015) Elucidating an affective pain circuit that creates a threat memory. Cell 162:363374. CrossRef Medline

Hou YY, Cai YQ, Pan ZZ (2015) Persistent pain maintains morphineseeking behavior after morphine withdrawal through reduced MeCP2 repression of GluA1 in rat central amygdala. J Neurosci 35:3689-3700. CrossRef Medline

Hunt SP, Mantyh PW (2001) The molecular dynamics of pain control. Nat Rev Neurosci 2:83-91. CrossRef Medline

Janak PH, Tye KM (2015) From circuits to behavior in the amygdala. Nature 517:284-292. CrossRef Medline

Kim J, Pignatelli M, Xu S, Itohara S, Tonegawa S (2016) Antagonistic negative and positive neurons of the basolateral amygdala. Nat Neurosci 19: 1636-1646. CrossRef Medline

Kitamura T, Ogawa SK, Roy DS, Okuyama T, Morrissey MD, Smith LM,
Redondo RL, Tonegawa S (2017) Engrams and circuits crucial for systems consolidation of a memory. Science 356:73-78. CrossRef Medline

Liu MG, Chen J (2014) Preclinical research on pain comorbidity with affective disorders and cognitive deficits: challenges and perspectives. Prog Neurobiol 116:13-32. CrossRef Medline

Lumley MA, Cohen JL, Borszcz GS, Cano A, Radcliffe AM, Porter LS, Schubiner H, Keefe FJ (2011) Pain and emotion: a biopsychosocial review of recent research. J Clin Psychol 67:942-968. CrossRef Medline

McWilliams LA, Cox BJ, Enns MW (2003) Mood and anxiety disorders associated with chronic pain: an examination in a nationally representative sample. Pain 106:127-133. CrossRef Medline

Micó JA, Ardid D, Berrocoso E, Eschalier A (2006) Antidepressants and pain. Trends Pharmacol Sci 27:348-354. CrossRef Medline

Murray EA (2007) The amygdala, reward and emotion. Trends Cogn Sci 11:489-497. CrossRef Medline

Navratilova E, Xie JY, Meske D, Qu C, Morimura K, Okun A, Arakawa N, Ossipov M, Fields HL, Porreca F (2015) Endogenous opioid activity in the anterior cingulate cortex is required for relief of pain. J Neurosci 35:7264-7271. CrossRef Medline

Neugebauer V (2015) Amygdala pain mechanisms. Handb Exp Pharmacol 227:261-284. CrossRef Medline

Paxinos G, Watson C (1986) The rat brain in stereotaxic coordinates, Ed 2. Sydney: Academic.

Price DD (2000) Psychological and neural mechanisms of the affective dimension of pain. Science 288:1769-1772. CrossRef Medline

Shackman AJ, Salomons TV, Slagter HA, Fox AS, Winter JJ, Davidson RJ (2011) The integration of negative affect, pain and cognitive control in the cingulate cortex. Nat Rev Neurosci 12:154-167. CrossRef Medline

Simons LE, Moulton EA, Linnman C, Carpino E, Becerra L, Borsook D (2014) The human amygdala and pain: evidence from neuroimaging. Hum Brain Mapp 35:527-538. CrossRef Medline

Slattery DA, Cryan JF (2012) Using the rat forced swim test to assess antidepressant-like activity in rodents. Nat Protoc 7:1009-1014. CrossRef Medline

Sugimura YK, Takahashi Y, Watabe AM, Kato F (2016) Synaptic and network consequences of monosynaptic nociceptive inputs of parabrachial nucleus origin in the central amygdala. J Neurophysiol 115:2721-2739. CrossRef Medline

Tye KM, Deisseroth K (2012) Optogenetic investigation of neural circuits underlying brain disease in animal models. Nat Rev Neurosci 13:251-266. CrossRef Medline

Tye KM, Prakash R, Kim SY, Fenno LE, Grosenick L, Zarabi H, Thompson KR, Gradinaru V, Ramakrishnan C, Deisseroth K (2011) Amygdala circuitry mediating reversible and bidirectional control of anxiety. Nature 471:358-362. CrossRef Medline

Vogt BA (2005) Pain and emotion interactions in subregions of the cingulate gyrus. Nat Rev Neurosci 6:533-544. CrossRef Medline

Wiech K, Tracey I (2009) The influence of negative emotions on pain: behavioral effects and neural mechanisms. Neuroimage 47:987-994. CrossRef Medline

Wilson KG, Mikail SF, D’Eon JL, Minns JE (2001) Alternative diagnostic criteria for major depressive disorder in patients with chronic pain. Pain 91:227-234. CrossRef Medline

Zald DH (2003) The human amygdala and the emotional evaluation of sensory stimuli. Brain Res Brain Res Rev 41:88-123. CrossRef Medline

Zhang Z, Cai YQ, Zou F, Bie B, Pan ZZ (2011) Epigenetic suppression of GAD65 expression mediates persistent pain. Nat Med 17:1448-1455. CrossRef Medline

Zhang Z, Tao W, Hou YY, Wang W, Kenny PJ, Pan ZZ (2014) MeCP2 repression of G9a in regulation of pain and morphine reward. J Neurosci 34:9076-9087. CrossRef Medline

Zhu W, Bie B, Pan ZZ (2007) Involvement of non-NMDA glutamate receptors in central amygdala in synaptic actions of ethanol and ethanolinduced reward behavior. J Neurosci 27:289-298. CrossRef Medline

Zhuo M (2016) Neural mechanisms underlying anxiety-chronic pain interactions. Trends Neurosci 39:136-145. CrossRef Medline 